\title{
GEMBASSY: an EMBOSS associated software package for comprehensive genome analyses
}

\author{
Hidetoshi Itaya ${ }^{1}$, Kazuki Oshita ${ }^{1,2}$, Kazuharu Arakawa ${ }^{1,2^{*}}$ and Masaru Tomita ${ }^{1,2}$
}

\begin{abstract}
The popular European Molecular Biology Open Software Suite (EMBOSS) currently contains over 400 tools used in various bioinformatics researches, equipped with sophisticated development frameworks for interoperability and tool discoverability as well as rich documentations and various user interfaces. In order to further strengthen EMBOSS in the fields of genomics, we here present a novel EMBOSS associated software (EMBASSY) package named GEMBASSY, which adds more than 50 analysis tools from the G-language Genome Analysis Environment and its Representational State Transfer (REST) and SOAP web services. GEMBASSY basically contains wrapper programs of G-language REST/SOAP web services to provide intuitive and easy access to various annotations within complete genome flatfiles, as well as tools for analyzing nucleic composition, calculating codon usage, and visualizing genomic information. For example, analysis methods such as for calculating distance between sequences by genomic signatures and for predicting gene expression levels from codon usage bias are effective in the interpretation of meta-genomic and meta-transcriptomic data. GEMBASSY tools can be used seamlessly with other EMBOSS tools and UNIX command line tools. The source code written in C is available from GitHub (https://github. com/celery-kotone/GEMBASSY/) and the distribution package is freely available from the GEMBASSY web site (http://www.g-language.org/gembassy/).
\end{abstract}

\section{Background}

First released in the year 2000, the European Molecular Biology Open Software Suite (EMBOSS) [1] is a comprehensive package for sequence analyses consisting of over 400 tools and is one of the most popular bioinformatics software packages. EMBOSS is not merely a collection of software tools, but is equipped with rich documentation and development framework to achieve high level of software interoperability and discoverability based on the Ajax Command Definitions (ACD) metadata for the tools. EMBOSS, therefore, is an interoperable bioinformatics software platform which work seamlessly in concert with other UNIX command-line tools, and it can alternatively be accessed from graphical user interface JEMBOSS [2] or from web based interface EMBOSS Explorer [3]. Third-party development using the EMBOSS platform is called the EMBOSS associated software (EMBASSY), and we have previously developed an EMBASSY package

\footnotetext{
* Correspondence: gaou@sfc.keio.ac.jp

${ }^{1}$ Institute for Advanced Biosciences, Keio University, 14-1, Baba town, Tsuruoka city, Yamagata Pref. 997-0035, Japan

${ }^{2}$ System Biology Program, Graduate School of Media and Governance, Keio

University, 5322, Endo, Fujisawa city, Kanagawa Pref. 252-0882, Japan
}

named the Keio Bioinformatics Web Service (KBWS) [4], which complements EMBOSS tools with access to 42 major bioinformatics web services such as NCBI BLAST and WebLogo. As a further expansion of EMBOSS, we hereby present a novel EMBASSY package designated GEMBASSY. This package adds over 50 tools for genome analysis and gene-centric sequence manipulation from genome flatfiles, implemented using methods from the Glanguage Genome Analysis Environment (G-language GAE) [5-7]. G-language GAE contains over 100 programs for genome analysis where most of which are implemented with published algorithms, and each of the programs are implemented with a variety of options and produces graphical output where available. Analysis programs included in the G-language GAE such as for the identification of conserved sequence motifs with information theory $[8,9]$, prediction of expression levels of genes from codon usage bias [10], visualization of GC skew [11] and prediction of replication origin and terminus [12,13], are effective in comparative study of bacterial genomes.

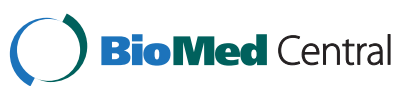

(c) 2013 Itaya et al.; licensee BioMed Central Ltd. This is an Open Access article distributed under the terms of the Creative Commons Attribution License (http://creativecommons.org/licenses/by/2.0), which permits unrestricted use, distribution, and reproduction in any medium, provided the original work is properly cited. 


\section{Implementation}

GEMBASSY is an EMBASSY package implemented on the EMBOSS platform and has 53 wrapper programs of the G-language web services [14]. The tools implemented in GEMBASSY are mainly consisted of whole genome analysis methods as listed in Table 1 (more detailed descriptions are given at the project web site: http://www.glanguage.org/gembassy/). Programs are named according to the corresponding G-language GAE method name prefixed with the letter "g". The software is written in $C$ with EMBOSS Ajax libraries, gSOAP Toolkit [15], and additional source code files created from the Glanguage SOAP Web Service Description Language (WSDL) file (http://soap.g-language.org/g-language. wsdl) using the wsdl2h utility included in the gSOAP Toolkit. GEMBASSY tools basically handles file input on the client side, and runs the actual analysis on the
G-language SOAP/REST server. By taking this clientserver architecture, the users do not need to install and maintain the G-language GAE, and can take advantage of the computational resource provided by the web services. Results of the analysis is then fetched by the client program, and output in EMBOSS-compliant manner. In order to expedite the transfer of large genome flatfiles, GEMBASSY provides the "-accid" option to specify and send only the accession ID of the sequence instead of transferring the entire information. Graphs are plotted using the EMBOSS graph plotting library, based on the Comma-Separated Values (CSV) file produced by the Glanguage web server, so that the image can be shown on screen through the X11 Window System or written out to 13 image formats supported in EMBOSS. The G-language SOAP service has been updated since its release to support accession ID as input and to support the latest

Table 1 Complete list of 53 tools implemented in GEMBASSY

\begin{tabular}{|c|c|c|c|}
\hline Category & Method name & Category & Method name \\
\hline \multirow[t]{2}{*}{ Data Retrieval } & gentrez & & gbasezvalue \\
\hline & genret & & gconsensusz \\
\hline \multirow[t]{5}{*}{ Display } & gcgr & & gdeltagcskew \\
\hline & gcircularmap & & gdistince \\
\hline & gdnawalk & & gfindoriter \\
\hline & ggenomemap3 & & ggcsi \\
\hline & gseq2png & & ggcskew \\
\hline \multirow[t]{14}{*}{ Nucleic Codon Usage } & gbui & & ggcwin \\
\hline & gcai & & ggeneskew \\
\hline & gcbi & & ggenomicskew \\
\hline & gcodoncompiler & & gkmertable \\
\hline & gdeltaenc & & gldabias \\
\hline & gdinuc & & gnucleotideperiodicity \\
\hline & genc & & goligomercounter \\
\hline & gew & & goligomersearch \\
\hline & gfop & & gpalindrome \\
\hline & gicdi & & gqueryarm \\
\hline & gp2 & & gquerystrand \\
\hline & gphx & & greporiter \\
\hline & gsvalue & & gscs \\
\hline & gwvalue & & gseqinfo \\
\hline \multirow[t]{6}{*}{ Nucleic Composition } & gb1 & & gsignature \\
\hline & gb2 & & gviewcds \\
\hline & gbasecounter & Nucleic Mutation & gshuffleseq \\
\hline & gbaseentropy & Protein Composition & gaminoinfo \\
\hline & gbaseinformationcontent & Protein Properties & gaaui \\
\hline & gbaserelativeentropy & & \\
\hline
\end{tabular}

Implemented tools are mostly consisted of genome analysis methods for nucleotide composition, codon usage, and genome information visualization, which are mainly implemented with published algorithms. The letter " $\mathrm{g}$ " is prefixed to indicate the software as a tool included in the GEMBASSY package. Detailed documentations for each of the tools as well as full references are available at the GEMBASSY web site (http://www.g-language.org/gembassy/). 
version of G-language GAE. The distribution package is available from the GEMBASSY web site (http:// www.g-language.org/gembassy/) or from GitHub reposi tory (https://github.com/celery-kotone/GEMBASSY) under the terms of GNU General Public License version 2. The EMBOSS Explorer interface is also available for use from the following URL (http://soap.g-language.org/gembassy/ emboss_explorer/).

\section{Results and discussion}

An example analysis workflows with GEMBASSY, several other EMBOSS commands, EMBASSY tools including KBWS, and UNIX command-line tools is shown in Figure 1. The workflow of Figure 1 studies a bacterial genome and analyzes the conservation of Shine-Dalgarno (SD) sequence by comparing the nucleotide composition in top and bottom 100 highly and lowly expressed genes. SD sequences are located directly upstream of start codons, and are recognized by the ribosome for translation initiation [16].

Here, the gene expression levels are predicted from synonymous codon usage bias based on the predicted highly expressed (PHX) index [17]. In this workflow, the accession ID of a genome of interest, which in this case is Bacillus subtilis [RefSeq:NC_000964], is searched in NCBI Entrez [18] using the gentrez utility of GEMBASSY (Figure 2-A). The flatfile of this genome is then retrieved using the seqret tool of EMBOSS. In order to study how SD sequence conservation is related to translation efficiency, the workflow next calculates the predicted gene expression level from the synonymous codon usage bias based on PHX (gphx tool). Using common UNIX commands sort, cut, head, and tail, top 100 highly expressed genes and bottom 100 lowly expressed genes are filtered as list of genes. Then, nucleotide sequences upstream of these genes are extracted from the genome flatfile using

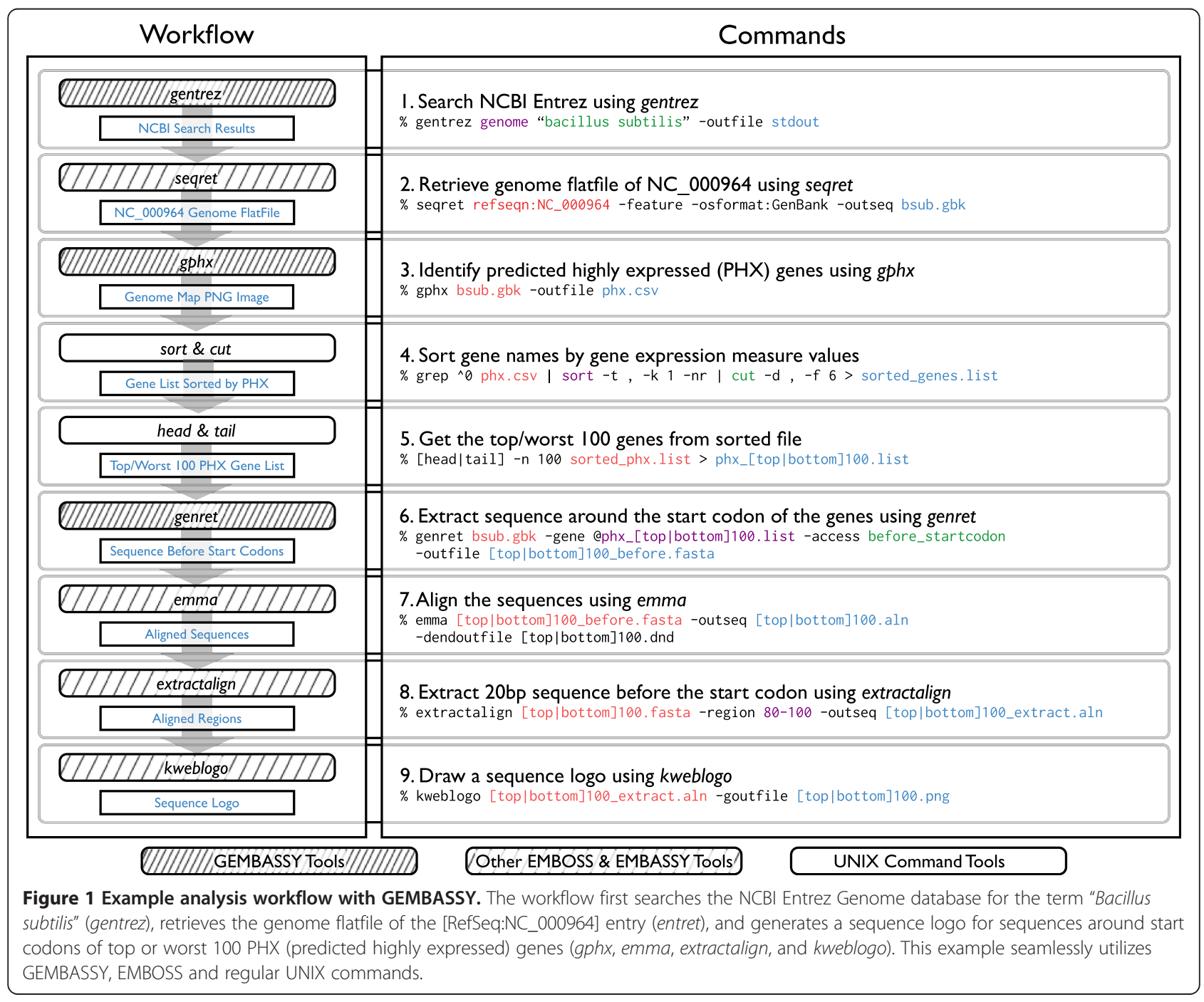



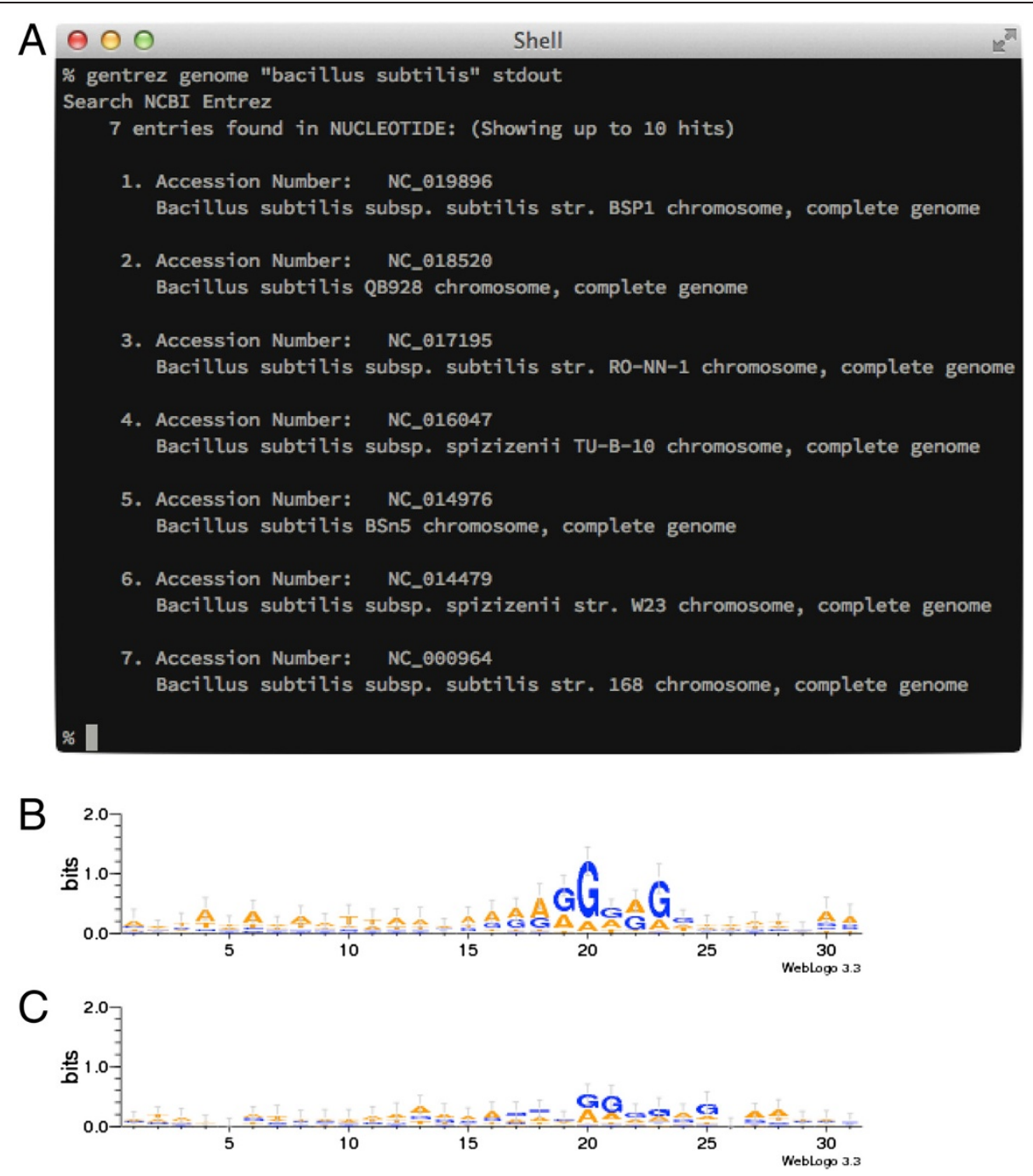

Figure 2 Graphical output from the sample workflow. (A) Result of keyword search in the NCBI Entrez Genome database with gentrez (result of Figure 1-1). (B and C) Sequence logos created with kweblogo, for top 100 (B) and worst 100 (C) PHX genes (Figure 1-9).

the genret utility. The genret tool is a versatile utility for the extraction of information in genome flatfiles, and users can easily retrieve feature information such as the gene product information and amino acid translation, as well as various nucleotide sequences such as those of the coding regions, exons, introns, or upstream and/or downstream regions. The emma utility is then used to align the sequences, and extractalign is used to extract the region of interest. Finally, these aligned upstream sequences are visualized using Sequence logos $[19,20]$ to compare the conservation levels of SD sequence. As shown in Figures 2-B and 2-C, top 100 PHX genes (Figure 2-B) show slightly higher conservation of SD sequence than the worst 100 PHX genes (Figure 2-C), suggesting lower translation initiation efficiency in genes that are lowly expressed. Three other sample workflows along with the example above are available online at our web site (http://www.g-language. org/gembassy/), with detailed information of the workflows available at the GEMBASSY web site.

As exemplified in the workflow, GEMBASSY complements existing EMBOSS/EMBASSY tools for the manipulation of genome flatfiles and adds numerous analysis tools suited for genome-level studies available in the G-language GAE. By making the tools available as an EMBASSY package based on EMBOSS framework, the users can use the same documentation ( $t \mathrm{fm}$ ) and discovery tools (wossname) of EMBOSS, and can take advantage of the familiar user interface that they are accustomed to.

\section{Abbreviations}

ACD: Ajax command definition; CSV: Comma-separated values;

EMBOSS: European Molecular Biology Open Software Suite; PHX: Predicted highly expressed; REST: Representational state transfer; SD: Shine-Dalgarno;

WSDL: Web service description language. 


\section{Competing interests}

The authors declare that they have no competing interests.

\section{Authors' contributions}

$\mathrm{HI}$ developed the software, KO updated the G-language SOAP service, $\mathrm{KO}$ and $\mathrm{KA}$ conceived of the project, and $\mathrm{HI}, \mathrm{KO}$, and $\mathrm{KA}$ designed the software and drafted the manuscript. MT supervised the project. All authors have read and approved the final manuscript.

\section{Acknowledgements}

This research was supported by funds from Yamagata Prefectural Government and Tsuruoka City, and by the KAKENHI Grant-in-Aid for Young Scientists (A), No.222681029.

Received: 31 March 2013 Accepted: 28 August 2013

Published: 29 August 2013

\section{References}

1. Rice $P$, Longden I, Bleasby A: EMBOSS: the European Molecular Biology Open Software Suite. Trends Genet 2000, 16:276-277.

2. Carver T, Bleasby A: The design of Jemboss: a graphical user interface to EMBOSS. Bioinformatics 2003, 19:1837-1843.

3. EMBOSS Explorer. http://embossgui.sourceforge.net/.

4. Oshita K, Arakawa K, Tomita M: KBWS: an EMBOSS associated package for accessing bioinformatics web services. Source Code Biol Med 2011, 6:8.

5. Arakawa K, Mori K, Ikeda K, Matsuzaki T, Kobayashi Y, Tomita M: G-language genome analysis environment: a workbench for nucleotide sequence data mining. Bioinformatics 2003, 19:305-306.

6. Arakawa K, Tomita M: G-language system as a platform for large-scale analysis of high-throughput omics data. J Pestic Sci 2006, 30:282-288.

7. Arakawa K, Suzuki H, Tomita M: Computational genome analysis using the G-language system. Genes Genomes Genomics 2008, 2:1-13.

8. Schneider TD: Measuring molecular information. J Theor Biol 1999, 201:87-92.

9. Schneider TD: Consensus sequence Zen. Applied bioinformatics 2002, 1:111-119.

10. Henry I, Sharp PM: Predicting gene expression level from codon usage bias. Mol Biol Evol 2007, 24:10-12.

11. Lobry JR: Asymmetric substitution patterns in the two DNA strands of bacteria. Mol Biol Evol 1996, 13:660-665.

12. Frank AC, Lobry JR: Oriloc: prediction of replication boundaries in unannotated bacterial chromosomes. Bioinformatics 2000, 16:560-561.

13. Arakawa K, Saito R, Tomita M: Noise-reduction filtering for accurate detection of replication termini in bacterial genomes. FEBS Lett 2007, 581:253-258.

14. Arakawa K, Kido N, Oshita K, Tomita M: G-language genome analysis environment with REST and SOAP web service interfaces. Nucleic Acids Res 2010, 38:W700-W705.

15. Van Engelen RA, Galliva KA: The gSOAP Toolkit for Web Services and Peer-to -Peer Computing Networks. Berlin: In Proceedings of the 2nd IEEE International Symposium on Cluster Computing and the Grid: 21-24 May 2002; 2002:128-135.

16. Shine J, Dalgarno L: The 3'-terminal sequence of Escherichia coli $16 \mathrm{~S}$ ribosomal RNA: complementarity to nonsense triplets and ribosome binding sites. Proc Natl Acad Sci U S A 1974, 71:1342-1346.

17. Karlin S, Mrazek J: Predicted highly expressed genes of diverse prokaryotic genomes. J Bacteriol 2000, 182:5238-5250.

18. Maglott D, Ostell J, Pruitt KD, Tatusova T: Entrez Gene: gene-centered information at NCBI. Nucleic Acids Res 2011, 39:D52-D57.

19. Schneider TD, Stephens RM: Sequence logos: a new way to display consensus sequences. Nucleic Acids Res 1990, 18:6097-6100.

20. Crooks GE, Hon G, Chandonia JM, Brenner SE: WebLogo: a sequence logo generator. Genome Res 2004, 14:1188-1190.

\section{Submit your next manuscript to BioMed Central and take full advantage of:}

- Convenient online submission

- Thorough peer review

- No space constraints or color figure charges

- Immediate publication on acceptance

- Inclusion in PubMed, CAS, Scopus and Google Scholar

- Research which is freely available for redistribution 